Bull. Korean Math. Soc. 48 (2011), No. 2, pp. 261-275

DOI 10.4134/BKMS.2011.48.2.261

\title{
DERIVATIVE OF THE RIESZ-NÁGY-TAKÁCS FUNCTION
}

\author{
IN-SOO BAEK
}

\begin{abstract}
We give characterizations of the differentiability points and the non-differentiability points of the Riesz-Nágy-Takács(RNT) singular function using the distribution sets in the unit interval. Using characterizations, we show that the Hausdorff dimension of the non-differentiability points of the RNT singular function is greater than 0 and the packing dimension of the infinite derivative points of the RNT singular function is less than 1. Further the RNT singular function is nowhere differentiable in the sense of topological magnitude, which leads to that the packing dimension of the non-differentiability points of the RNT singular function is 1 . Finally we show that our characterizations generalize a recent result from the $(\tau, \tau-1)$-expansion associated with the RNT singular function adding a new result for a sufficient condition for the non-differentiability points.
\end{abstract}

\section{Introduction}

Recently many authors $([6,7,8])$ studied non-differentiability and some characterization of the non-differentiability set of a Cantor function. They used a fractal method to find the characterization. The Cantor function is a singular function, which is not strictly increasing. More recently J. Paradís et al. ([14]) studied some conditions of the null and infinite derivatives of the RNT strictly increasing singular function using their so-called $(\tau, \tau-1)$-expansions of the unit interval. In this paper, we also study the conditions of the null and infinite derivatives of the singular function using a multifractal method which is easier than the direct calculation of derivatives using $(\tau, \tau-1)$-expansions of the unit interval in some sense. Further our multifractal method gives more information than the $(\tau, \tau-1)$-expansion method.

We can express any point in $(0,1]$ as a code using the generalized dyadic expansion with a positive real number base $a \in(0,1)([2])$. We define a strictly increasing function $f$ which corresponds each code with a base $a$ to the same code with a base $p \in(0,1)$. We call such a function $f$ the base transformed identical code function. In fact, the base transformed identical code function $f$

Received June 1, 2009.

2010 Mathematics Subject Classification. 26A30, 28A80.

Key words and phrases. Hausdorff dimension, packing dimension, distribution set, local dimension set, singular function, metric number theory. 
is the RNT function. We show that $0<f^{\prime}(x)<\infty$ on a subset of Hausdorff dimension less than 1 when $p \neq a$, which leads to that the derivative $f^{\prime}(x)$ at almost every point $x$ in the unit interval is 0 . This means that when $p \neq a$ the function $f$ is a singular function.

The distribution sets in the unit interval are the local dimension sets by a self-similar measure ([2]). We note that the Hausdorff and packing dimensions of the local dimension sets in the unit interval were obtained in [2] by the cylinder density theorem $([5,11])$ instead of the usual density theorem $([10])$. In fact, the local dimension set relates $p$ with $a$ in the RNT function $f$.

Using the information of the distribution sets and the local dimension sets, we give some multifractal characterizations of the derivative sets and the nondifferentiability set of $f$. As a result, we see that the RNT singular function $f$ is differentiable only on a meager subset of the unit interval. Further the Hausdorff dimension of the non-differentiability set of the RNT singular function $f$ is greater 0 whereas its packing dimension is 1 and the packing dimension of the infinite derivative set of the RNT singular function $f$ is less than 1 .

Finally we show that our multifractal characterization is a generalization of a recent result ([14]) for the RNT singular function. Consequently our multifractal characterization gives surprisingly rich information about $(\tau, \tau-1)$ expansions associated with the RNT singular function. That is, the solution $r=r(1)$ of the equation $\left(\frac{p}{a}\right)^{r}\left(\frac{1-p}{1-a}\right)^{1-r}=1$ gives a bifurcation point for its null and infinite derivative whereas for $\alpha=1 / a, \tau=1 / p$

$$
K=K(\alpha, \tau)=\frac{\log \left(\frac{\alpha-1}{\tau-1}\right)}{\log (\alpha / \tau)}\left(=\frac{1}{1-r(1)}\right)
$$

with respect to the asymptotic behavior of $\frac{a_{n}}{n}$ from the $(\tau, \tau-1)$-expansion gives a critical point for its derivative (see our Section 4 or [14]). Further our application to the critical point $K$ adds new facts of a sufficient condition for the non-differentiability points of the RNT singular distribution and the converse of the main theorem in [14].

\section{Preliminaries}

We (cf. [2]) define $F_{a}$ the unit interval $(0,1]$ having the generalized dyadic expansion with a base $a$ where $0<a<1$. Let $\mathbb{N}$ be the set of the positive integers. We define a fundamental interval $I_{i_{1} \cdots i_{k}}=f_{i_{1}} \circ \cdots \circ f_{i_{k}}(I)$ where $f_{0}(x)=a x$ and $f_{1}(x)=(1-a) x+a$ on $I=(0,1], i_{j} \in\{0,1\}$ and $1 \leq$ $j \leq k$. If $x \in F_{a}=(0,1]$, then there is a unique code $\sigma \in\{0,1\}^{\mathbb{N}}$ such that $\bigcap_{k=1}^{\infty} I_{\sigma \mid k}=\{x\}$ (Here $\sigma \mid k=i_{1} i_{2} \cdots i_{k}$ where $\left.\sigma=i_{1} i_{2} \cdots i_{k} i_{k+1} \cdots\right)$. We call a code $\sigma \in\{0,1\}^{\mathbb{N}}$ where $\bigcap_{k=1}^{\infty} I_{\sigma \mid k}=\{x\}$ the generalized dyadic expansion with $a$ base $a$ of $x$ and identify $x$ with the code $\sigma$ without confusion. We write $[\sigma]_{(a)}$ for the generalized dyadic expansion with a base $a$ of $x$ to avoid some confusion. We use non-terminating expression for the end points of the fundamental intervals whereas we used terminating expression for them in [2]. 
In this sense, we can still use the results of [2] in our paper. In fact, we use this non-terminating expression for the expansion to be compatible with the $(\tau, \tau-1)$ expansion for the RNT function.

We note that $F_{\frac{1}{2}}$ is the unit interval $(0,1]$ having the dyadic expansion (cf. [12]). If $x \in F_{a}=(0,1]$ and $x \in I_{\sigma^{\prime}}$ where $\sigma^{\prime} \in\{0,1\}^{k}$, a cylinder $c_{k}(x)$ denotes the fundamental interval $I_{\sigma^{\prime}}$ and $\left|c_{k}(x)\right|$ denotes the diameter of $c_{k}(x)$ for each $k=0,1,2, \ldots$. We define the base transformed identical code function

$$
f: F_{a} \rightarrow F_{p}
$$

such that $f\left([\sigma]_{(a)}\right)=[\sigma]_{(p)}$ where $0<a, p<1$. More precisely, $f$ carries $x\left(=[\sigma]_{(a)} \in(0,1]\right)$ which has the generalized dyadic expansion $\sigma$ with a base $a$ to $y\left(=[\sigma]_{(p)} \in(0,1]\right)$ which has the same code $\sigma$ as its generalized dyadic expansion with a base $p$. Then we easily see that it is a continuous strictly increasing function from $(0,1]$ onto $(0,1]$. If $p=a$, then the above function $f(x)$ is the identity function which is a trivial case for consideration. It is not difficult to show that it is the Riesz-Nágy-Takács(RNT) function ([14]), that is, $f(x)=\Phi_{\alpha, \tau}(x)$ where $\alpha=\frac{1}{a}$ and $\tau=\frac{1}{p}$. Precisely, given $\alpha, \tau>1$ and $x \in(0,1]$,

$$
\Phi_{\alpha, \tau}(x)=\sum_{j=1}^{\infty} \frac{(\tau-1)^{j-1}}{\tau^{a_{j}}}
$$

for

$$
x=\sum_{j=1}^{\infty} \frac{(\alpha-1)^{j-1}}{\alpha^{a_{j}}}
$$

with integers $1 \leq a_{1}<a_{2}<\cdots<a_{n}<\cdots$. In this case, we note that J. Paradís et al. used a non-terminating expansion and the above $x$ can be represented in our code by

$$
x=\overbrace{0 \cdots 0}^{a_{1}-1} 1 \overbrace{0 \cdots 0}^{a_{2}-a_{1}-1} 1 \cdots \overbrace{0 \cdots 0}^{a_{n}-a_{n-1}-1} 1 \cdots .
$$

That is, $a_{j}$ means the $j$-th position of 1 in our code. For example, $a=$ $\sum_{j=1}^{\infty} \frac{(\alpha-1)^{j-1}}{\alpha^{j+1}}$ and $a_{j}=j+1$ with $a=0111 \cdots$ in our code. For another example, if $x=\sum_{j=1}^{\infty} \frac{(\alpha-1)^{j-1}}{\alpha^{2 j-1}}$, then $a_{j}=2 j-1$ with $x=101010 \cdots$ in our code. We note that their expression for $x \in(0,1]$ are exactly the same as our codes essentially.

Remark 1 . Let $f:(0,1] \rightarrow(0,1]$ be a strictly increasing function from $(0,1]$ onto $(0,1]$. Since every real valued increasing function has its derivative at almost every point in $(0,1]$ ([15]) (from now on, almost everywhere means Lebesgue measure almost everywhere),

$$
0 \leq f^{\prime}(x)<\infty .
$$

However, we say that $f^{\prime}(x)$ exists in a wide sense if $f^{\prime}(x)$ exists and $0 \leq f^{\prime}(x) \leq$ $\infty$ (cf. [14]). 
From now on $\operatorname{dim}(E)$ denotes the Hausdorff dimension of $E$ and $\operatorname{Dim}(E)$ denotes the packing dimension of $E([10])$. We note that $\operatorname{dim}(E) \leq \operatorname{Dim}(E)$ for every set $E([10])$. We denote $n_{0}(x \mid k)$ the number of times the digit 0 occurs in the first $k$ places of $x=\sigma$ (cf. [1]). In $F_{a}$, for $r \in[0,1]$, we define the lower(upper) distribution set $\underline{F}(r)(\bar{F}(r))$ containing the digit 0 in proportion $r$ by

$$
\begin{aligned}
& \underline{F}(r)=\left\{x \in(0,1]: \liminf _{k \rightarrow \infty} \frac{n_{0}(x \mid k)}{k}=r\right\}, \\
& \bar{F}(r)=\left\{x \in(0,1]: \limsup _{k \rightarrow \infty} \frac{n_{0}(x \mid k)}{k}=r\right\} .
\end{aligned}
$$

We write $\underline{F}(r) \cap \bar{F}(r)=F(r)$ and call it the distribution set containing the digit 0 in proportion $r$. Let $p \in(0,1)$ and denote $\gamma_{p}$ a self-similar Borel probability measure on $(0,1]$ satisfying $\gamma_{p}\left(I_{0}\right)=p($ cf. $[1,10])$. We write $\underline{E}_{\alpha}^{(p)}$ $\left(\bar{E}_{\alpha}^{(p)}\right)$ for the set of points at which the lower(upper) local cylinder density of $\gamma_{p}$ on $(0,1]$ is exactly $\alpha$, so that

$$
\begin{aligned}
& \underline{E}_{\alpha}^{(p)}=\left\{x \in(0,1]: \liminf _{k \rightarrow \infty} \frac{\log \gamma_{p}\left(c_{k}(x)\right)}{\log \left|c_{k}(x)\right|}=\alpha\right\}, \\
& \bar{E}_{\alpha}^{(p)}=\left\{x \in(0,1]: \limsup _{k \rightarrow \infty} \frac{\log \gamma_{p}\left(c_{k}(x)\right)}{\log \left|c_{k}(x)\right|}=\alpha\right\} .
\end{aligned}
$$

We write $\underline{E}_{\alpha}^{(p)} \cap \bar{E}_{\alpha}^{(p)}=E_{\alpha}^{(p)}$ and call it the local dimension set having local dimension $\alpha$ by a self-similar measure $\gamma_{p}$. In this paper, we assume that $0 \log 0=0$ for convenience. We introduce the relation between the distribution sets and the local dimension sets, which is an essential result from [1].

Proposition 1. Let $p \in(0,1), r \in[0,1]$ and $g(r, p)=\frac{r \log p+(1-r) \log (1-p)}{r \log a+(1-r) \log (1-a)}$. Then in $F_{a}=(0,1]$,

(1) $\underline{F}(r)=\underline{E}_{g(r, p)}^{(p)}$ if $0<p<a$,

(2) $\underline{F}(r)=\bar{E}_{g(r, p)}^{(p)}$ if $a<p<1$,

(3) $\bar{F}(r)=\bar{E}_{g(r, p)}^{(p)}$ if $0<p<a$,

(4) $\bar{F}(r)=\underline{E}_{g(r, p)}^{(p)}$ if $a<p<1$.

Proof. It follows from the same arguments of the proof of the Theorems 2 in $[1,2]$.

From now on, we will continue to use $g(r, p)$ as above.

\section{Characterizations of the derivative sets and the non-differentiability set}

Lemma 2. Let $\left\{\alpha_{n}\right\}$ and $\left\{\alpha_{n}^{\prime}\right\}$ be positive real sequences and $\left\{\beta_{n}\right\}$ and $\left\{\beta_{n}^{\prime}\right\}$ be real sequences. If $\lim _{n \rightarrow \infty} \frac{\beta_{n}}{\alpha_{n}}=\lim _{n \rightarrow \infty} \frac{\beta_{n}^{\prime}}{\alpha_{n}^{\prime}}=c$ for some real number $c$, then $\lim _{n \rightarrow \infty} \frac{\beta_{n}+\beta_{n}^{\prime}}{\alpha_{n}+\alpha_{n}^{\prime}}=c$. 
Proof. Noting that $\alpha_{n}+\alpha_{n}^{\prime}>0$, we easily obtain the result.

Lemma 3. Let $f:(0,1] \longrightarrow(0,1]$ be a differentiable function at a point $x \in(0,1)$. Then the derivative $f^{\prime}(x)$ of $f$ at $x$ satisfies the following condition:

$$
\lim _{n \rightarrow \infty} \frac{f\left(z_{n}\right)-f\left(y_{n}\right)}{z_{n}-y_{n}}=f^{\prime}(x)
$$

where $y_{n} \uparrow x$ and $z_{n} \downarrow x$.

Proof. Since $f^{\prime}(x)$ exists and its value is a real number, we clearly see that

$$
\lim _{y \uparrow x} \frac{f(x)-f(y)}{x-y}=\lim _{z \downarrow x} \frac{f(z)-f(x)}{z-x}=f^{\prime}(x) .
$$

For all sequences $\left\{y_{n}\right\}$ and $\left\{z_{n}\right\}$ such that $y_{n} \uparrow x$ and $z_{n} \downarrow x$, we also see that

$$
\lim _{n \rightarrow \infty} \frac{f(x)-f\left(y_{n}\right)}{x-y_{n}}=\lim _{n \rightarrow \infty} \frac{f\left(z_{n}\right)-f(x)}{z_{n}-x}=f^{\prime}(x) .
$$

It follows from the above lemma.

Theorem 4. Let $f: F_{a} \rightarrow F_{p}$ be the base transformed identical code function such that $f\left([\sigma]_{(a)}\right)=[\sigma]_{(p)}$ where $0<p<1$. Then the derivative $f^{\prime}(x)$ of $f$ at $x$ exists for almost every point $x \in(0,1]$. Further it satisfies the following condition:

$$
0 \leq \lim _{n \rightarrow \infty} \frac{\gamma_{p}\left(c_{n}(x)\right)}{\left|c_{n}(x)\right|}=f^{\prime}(x)<\infty
$$

where $\gamma_{p}$ is a self-similar probability measure on $(0,1]$ satisfying $\gamma_{p}\left(I_{0}\right)=p$ and $p \in(0,1)$.

Proof. Since $f$ is a strictly increasing function from $(0,1]$ onto $(0,1]$, the derivative $f^{\prime}(x)$ of $f$ at $x$ exists for almost every point $x \in(0,1]$ (Remark 1 ). If we put $c_{n}(x)=\left(y_{n}, z_{n}\right]$, then we clearly see that $\gamma_{p}\left(c_{n}(x)\right)=f\left(z_{n}\right)-f\left(y_{n}\right)$. To apply this to the above lemma, we should exclude the point $x$ such that $x=y_{n}$ or $x=z_{n}$. Such $x$ is only an end point of a fundamental interval. It follows from the above lemma since the derivative $f^{\prime}(x)$ of $f$ at $x$ does not exist for an end point $x$ of a fundamental interval.

Theorem 5. Let $f: F_{a} \rightarrow F_{p}$ be the base transformed identical code function such that $f\left([\sigma]_{(a)}\right)=[\sigma]_{(p)}$. For $x \in(0,1]$ at which $f^{\prime}(x)$ exists and $0<f^{\prime}(x)<$ $\infty$,

$$
\lim _{n \rightarrow \infty} \frac{\log \gamma_{p}\left(c_{n}(x)\right)}{\log \left|c_{n}(x)\right|}=1 .
$$

Proof. Since log function is a continuous function,

$$
\lim _{n \rightarrow \infty} \log \frac{\gamma_{p}\left(c_{n}(x)\right)}{\left|c_{n}(x)\right|}=\log f^{\prime}(x) .
$$

It follows easily since $0<f^{\prime}(x)<\infty$. 
Theorem 6. Let $f: F_{a} \rightarrow F_{p}$ be the base transformed identical code function such that $f\left([\sigma]_{(a)}\right)=[\sigma]_{(p)}$. Assume that $p \neq a$. Then

$$
\left\{x \in(0,1]: 0<f^{\prime}(x)<\infty\right\} \subset E_{1}^{(p)}
$$

with $\operatorname{dim}\left(E_{1}^{(p)}\right)<1$, hence the derivative $f^{\prime}(x)=0$ for almost every point $x \in(0,1]$.

Proof. From the above theorem, if $x \in(0,1]$ at which $f^{\prime}(x)$ exists and $0<$ $f^{\prime}(x)<\infty$ then such $x \in E_{1}^{(p)}$. Since $p \neq a$, we have $r \neq a$ when $g(r, p)=1$, which gives $g(r, r)<1$. From Corollary 7 (1) in [2], we have

$$
\operatorname{dim}\left(E_{1}^{(p)}\right)=g(r, r)<1,
$$

where $E_{1}^{(p)} \subset F_{a}=(0,1]$. This means that the Lebesgue measure of the set $E_{1}^{(p)}$ is 0 . It follows from that $0 \leq f^{\prime}(x)<\infty$ for almost every point $x \in(0,1]$ from Remark 1.

Remark 2. P. Billingsley ([4]) essentially showed that $\left\{x \in(0,1]: 0<f^{\prime}(x)<\right.$ $\infty\}=\phi$ using the fact that

$$
\lim _{n \rightarrow \infty}\left|\frac{\gamma_{p}\left(c_{n+1}(x)\right)}{\gamma_{p}\left(c_{n}(x)\right)}-\frac{\left|c_{n+1}(x)\right|}{\left|c_{n}(x)\right|}\right|=0
$$

for $x \in\left\{x \in(0,1]: 0<f^{\prime}(x)<\infty\right\}$ whereas $\left|\frac{\gamma_{p}\left(c_{n+1}(x)\right)}{\gamma_{p}\left(c_{n}(x)\right)}-\frac{\left|c_{n+1}(x)\right|}{\left|c_{n}(x)\right|}\right|=|p-a| \neq$ 0 . We give our multifractal spectrum method as an alternative of Billingsley's direct method to show that $f$ is a singular function.

From now on, without any additional condition we assume that $f: F_{a} \rightarrow F_{p}$ is the base transformed identical code function such that $f\left([\sigma]_{(a)}\right)=[\sigma]_{(p)}$ where $0<p(\neq a)<1$ and $r(1)$ denotes the solution $r$ of the equation $g(r, p)=$ 1 .

Remark 3. Let $N=(0,1]-\left(D_{0} \cup D_{\infty} \cup D_{1}\right)$ where

$$
\begin{aligned}
D_{0} & =\left\{x \in(0,1]: f^{\prime}(x)=0\right\}, \\
D_{\infty} & =\left\{x \in(0,1]: f^{\prime}(x)=\infty\right\}, \\
D_{1} & =\left\{x \in(0,1]: 0<f^{\prime}(x)<\infty\right\} .
\end{aligned}
$$

Then $N=(0,1]-\left(D_{0} \cup D_{\infty}\right)$ since $D_{1}=\phi$ from Remark 2. Further $N$ is the set of the points $x$ at which the derivatives $f^{\prime}(x)$ of $f$ do not exist. As we mentioned earlier, the end points of any fundamental dyadic interval are in $N$. From now on, we will continue to use $D_{0}, D_{\infty}, N$ as above.

We give some characterizations of the points $x$ where $f^{\prime}(x)=0$ and $f^{\prime}(x)=$ $\infty$ respectively when $a<p<1$ using distribution sets.

Theorem 7. Let $a<p<1$. Then

$$
D_{0} \subset \cup_{0 \leq r \leq r(1)} \underline{F}(r) \text { and } D_{\infty} \subset \cup_{r(1) \leq r \leq 1} \bar{F}(r) \text {. }
$$


Proof. We note that $\liminf _{n \rightarrow \infty} \frac{\log \gamma_{p}\left(c_{n}(x)\right)}{\log \left|c_{n}(x)\right|}>1$ implies $\lim _{n \rightarrow \infty} \frac{\gamma_{p}\left(c_{n}(x)\right)}{\left|c_{n}(x)\right|}=$ 0 . It is not difficult to show that $g(r, p)$ is a decreasing function for $r$ when $a<p<1$. Since $\underline{E}_{g(r(1), p)}^{(p)}=\underline{E}_{1}^{(p)}$ and $g(r, p)$ is a decreasing function for $r$, if $x \in \underline{E}_{g(r, p)}^{(p)}$ where $0 \leq r<r(1)$, then $\lim _{n \rightarrow \infty} \frac{\gamma_{p}\left(c_{n}(x)\right)}{\left|c_{n}(x)\right|}=0$. Using its contraposition and Lemma 3 , we easily see that $D_{\infty} \subset \cup_{r(1) \leq r \leq 1} \underline{E}_{g(r, p)}^{(p)}$. It follows from that $\underline{E}_{g(r, p)}^{(p)}=\bar{F}(r)$ from Proposition 1. Similarly we note that $\limsup _{n \rightarrow \infty} \frac{\log \gamma_{p}\left(c_{n}(x)\right)}{\log \left|c_{n}(x)\right|}<1$ implies $\lim _{n \rightarrow \infty} \frac{\gamma_{p}\left(c_{n}(x)\right)}{\left|c_{n}(x)\right|}=\infty$. Therefore if $x \in \bar{E}_{g(r, p)}^{(p)}$ where $r(1)<r \leq 1$, then $\lim _{n \rightarrow \infty} \frac{\gamma_{p}\left(c_{n}(x)\right)}{\left|c_{n}(x)\right|}=\infty$. Using its contraposition and Lemma 3 , we easily see that $D_{0} \subset \cup_{0 \leq r \leq r(1)} \bar{E}_{g(r, p)}^{(p)}$. It follows from that $\bar{E}_{g(r, p)}^{(p)}=\underline{F}(r)$ from Proposition 1 .

Dually we easily see the similar results for the points $x$ where $f^{\prime}(x)=0$ and $f^{\prime}(x)=\infty$ respectively when $0<p<a$ using distribution sets.

Theorem 8. Let $0<p<a$. Then

$$
D_{0} \subset \cup_{r(1) \leq r \leq 1} \bar{F}(r) \text { and } D_{\infty} \subset \cup_{0 \leq r \leq r(1)} \underline{F}(r) .
$$

Proof. It is not difficult to show that $g(r, p)$ is an increasing function for $r$ when $0<p<a$. It follows from the dual arguments of the proof of the above theorem.

To avoid a degeneration case, we exclude the end points of the fundamental intervals for the following two theorems.

Theorem 9. Assume that $x$ is not an end point of a fundamental interval. If $\lim _{n \rightarrow \infty} \frac{\gamma_{p}\left(c_{n}(x)\right)}{\left|c_{n}(x)\right|}=0$, then

$$
\lim _{n \rightarrow \infty} \frac{f(x)-f\left(y_{n}\right)}{x-y_{n}}=0=\lim _{n \rightarrow \infty} \frac{f\left(z_{n}\right)-f(x)}{z_{n}-x},
$$

where $y_{n}$ is the left end point of $c_{n}(x)$ and $z_{n}$ is the right end point of $c_{n}(x)$. Similarly if $\lim _{i \rightarrow \infty} \frac{\gamma_{p}\left(c_{n_{i}}(x)\right)}{\left|c_{n_{i}}(x)\right|}=0$, then

$$
\lim _{i \rightarrow \infty} \frac{f(x)-f\left(y_{n_{i}}\right)}{x-y_{n_{i}}}=0=\lim _{i \rightarrow \infty} \frac{f\left(z_{n_{i}}\right)-f(x)}{z_{n_{i}}-x},
$$

where $y_{n_{i}}$ is the left end point of $c_{n_{i}}(x)$ and $z_{n_{i}}$ is the right end point of $c_{n_{i}}(x)$.

Proof. We note that $x$ has the generalized dyadic expansion with a base $a$ where $0<a<1$. Suppose that it does not hold that $\lim _{n \rightarrow \infty} \frac{f(x)-f\left(y_{n}\right)}{x-y_{n}}=0$. Then for some $\epsilon>0$ there are infinitely many $n$ such that $\frac{f(x)-f\left(y_{n}\right)}{x-y_{n}}>\epsilon$. We may assume that $\frac{f(x)-f\left(y_{n_{k}}\right)}{x-y_{n_{k}}}>\epsilon$ for all $k \in \mathbb{N}$ for some subsequence $\left\{y_{n_{k}}\right\}$ of distinct members such that $y_{n_{1}}<y_{n_{2}}<\cdots<y_{n_{k}}<y_{n_{k+1}}<\cdots$ of the sequence $\left\{y_{n}\right\}$ since $x$ is not an end point of a fundamental interval. 
Assume that $\lim _{k \rightarrow \infty} \frac{f(x)-f\left(y_{n_{k}}\right)}{z_{n_{k}}-y_{n_{k}}} \neq 0$. Then we easily see that there exists a subsequence $\left\{y_{n_{k j}}\right\}$ of the sequence $\left\{y_{n_{k}}\right\}$ and $\frac{f(x)-f\left(y_{n_{k_{j}}}\right)}{z_{n_{k_{j}}}-y_{n_{k_{j}}}}>\eta$ for some $\eta>0$ and for all $j \in \mathbb{N}$. Hence $\eta \leq \frac{f\left(z_{n_{k j}}\right)-f\left(y_{n_{k j}}\right)}{z_{n_{k j}}-y_{n_{k j}}}=\frac{\gamma_{p}\left(c_{n_{k j}}(x)\right)}{\left|c_{n_{k j}}(x)\right|}$ for all $j \in \mathbb{N}$, which is a contradiction. Hence $\lim _{k \rightarrow \infty} \frac{f(x)-f\left(y_{n_{k}}\right)}{z_{n_{k}}-y_{n_{k}}}=0$. Therefore for $\delta$ such that $0<\delta<\frac{a \epsilon}{2}$, there exists an integer $M$ such that $0<\frac{f(x)-f\left(y_{n_{k}}\right)}{z_{n_{k}}-y_{n_{k}}}<\delta$ for all $k \geq M$. Since $y_{n_{k}} \uparrow x$ and $y_{n_{1}}<y_{n_{2}}<\cdots<y_{n_{k}}<y_{n_{k+1}}<\cdots$, we can find

$$
i_{k}=\max \left\{i \in \mathbb{N} \cup\{0\}: y_{n_{k}}=\cdots=y_{n_{k}+i}\right\} .
$$

Let $m_{k}=n_{k}+i_{k}$. Then $\frac{\left|c_{m_{k}}(x)\right|}{x-y_{m_{k}}} \leq \frac{a^{i}(1-a)^{m_{k}-i}}{a^{i}(1-a)^{m_{k}-i} a}=\frac{1}{a}$ where $\left|c_{m_{k}}(x)\right|=$ $a^{i}(1-a)^{m_{k}-i}$ for some integer $i$. Suppose that $\lim _{k \rightarrow \infty} \frac{f(x)-f\left(y_{m_{k}}\right)}{z_{m_{k}}-y_{m_{k}}}=0$. Then there exists an integer $L \geq M$ such that $0<\frac{f(x)-f\left(y_{m_{k}}\right)}{z_{m_{k}}-y_{m_{k}}}<\delta$ for all $k \geq L$. Since $y_{m_{k}}=y_{n_{k}}$, we easily see that $\epsilon\left(x-y_{m_{k}}\right)<f(x)-f\left(y_{m_{k}}\right)<\delta\left(z_{m_{k}}-y_{m_{k}}\right)$ for all $k \geq L$. Since $z_{m_{k}}-y_{m_{k}}=\left|c_{m_{k}}(x)\right|, \frac{2}{a}<\frac{\epsilon}{\delta}<\frac{\left|c_{m_{k}}(x)\right|}{x-y_{m_{k}}} \leq \frac{1}{a}$ for all $k \geq L$, which is a contradiction. Since $\lim _{k \rightarrow \infty} \frac{f(x)-f\left(y_{m_{k}}\right)}{z_{m_{k}}-y_{m_{k}}} \neq 0$, we also have a subsequence $\left\{y_{m_{k_{j}}}\right\}$ of the sequence $\left\{y_{m_{k}}\right\}$ such that $\frac{f\left(z_{m_{k j}}\right)-f\left(y_{m_{k j}}\right)}{z_{m_{k j}}-y_{m_{k_{j}}}}=$ $\frac{\gamma_{p}\left(c_{m_{k j}}(x)\right)}{\left|c_{m_{k j}}(x)\right|} \geq \eta^{\prime}>0$ for some $\eta^{\prime}>0$ and for all $j \in \mathbb{N}$, which gives a contradiction. $\lim _{n \rightarrow \infty} \frac{f\left(z_{n}\right)-f(x)}{z_{n}-x}=0$ follows from the similar arguments. The subsequential result also holds from the same arguments above.

Theorem 10. Assume that $x$ is not an end point of a fundamental interval. If $\lim _{n \rightarrow \infty} \frac{\gamma_{p}\left(c_{n}(x)\right)}{\left|c_{n}(x)\right|}=\infty$, then

$$
\lim _{n \rightarrow \infty} \frac{f(x)-f\left(y_{n}\right)}{x-y_{n}}=\infty=\lim _{n \rightarrow \infty} \frac{f\left(z_{n}\right)-f(x)}{z_{n}-x},
$$

where $y_{n}$ is the left end point of $c_{n}(x)$ and $z_{n}$ is the right end point of $c_{n}(x)$. Similarly if $\lim _{i \rightarrow \infty} \frac{\gamma_{p}\left(c_{n_{i}}(x)\right)}{\left|c_{n_{i}}(x)\right|}=\infty$, then

$$
\lim _{i \rightarrow \infty} \frac{f(x)-f\left(y_{n_{i}}\right)}{x-y_{n_{i}}}=\infty=\lim _{i \rightarrow \infty} \frac{f\left(z_{n_{i}}\right)-f(x)}{z_{n_{i}}-x},
$$

where $y_{n_{i}}$ is the left end point of $c_{n_{i}}(x)$ and $z_{n_{i}}$ is the right end point of $c_{n_{i}}(x)$.

Proof. It follows from the same arguments of the proof of the above theorem.

The following two corollaries are our characterizations of the null derivative set, the infinite derivative set and the non-differentiability set of the RNT singular function using distribution sets. 


\section{Corollary 11.}

$$
\left[\cup_{r(1)<r \leq 1} \bar{F}(r)\right] \cap\left[\cup_{0 \leq r<r(1)} \underline{F}(r)\right] \subset N .
$$

Proof. We only need to show that $f^{\prime}(x)$ does not exist for

$$
x \in\left[\cup_{r(1)<r \leq 1} \bar{F}(r)\right] \cap\left[\cup_{0 \leq r<r(1)} \underline{F}(r)\right] .
$$

It is not difficult to show that

$$
\liminf _{n \rightarrow \infty} \frac{\log \gamma_{p}\left(c_{n}(x)\right)}{\log \left|c_{n}(x)\right|}<1
$$

and

$$
\limsup _{n \rightarrow \infty} \frac{\log \gamma_{p}\left(c_{n}(x)\right)}{\log \left|c_{n}(x)\right|}>1
$$

for

$$
x \in\left[\cup_{r(1)<r \leq 1} \bar{F}(r)\right] \cap\left[\cup_{0 \leq r<r(1)} \underline{F}(r)\right]
$$

from Proposition 1. This implies that

$$
\limsup _{n \rightarrow \infty} \frac{\gamma_{p}\left(c_{n}(x)\right)}{\left|c_{n}(x)\right|}=\infty
$$

and

$$
\liminf _{n \rightarrow \infty} \frac{\gamma_{p}\left(c_{n}(x)\right)}{\left|c_{n}(x)\right|}=0
$$

Since $x$ is not an end point of a fundamental interval, it follows from the subsequential results of the above two theorems.

Corollary 12. For $a<p<1$,

$$
\begin{aligned}
& \cup_{0 \leq r \leq r(1)} \bar{F}(r)-F(r(1)) \subset D_{0} \cup N, \\
& \cup_{r(1) \leq r \leq 1} \underline{F}(r)-F(r(1)) \subset D_{\infty} \cup N,
\end{aligned}
$$

further

$$
\begin{aligned}
& D_{0} \subset \cup_{0 \leq r \leq r(1)} \bar{F}(r), \\
& D_{\infty} \subset \cup_{r(1) \leq r \leq 1} \underline{F}(r) .
\end{aligned}
$$

Similarly for $0<p<a$,

$$
\begin{aligned}
& \cup_{r(1) \leq r \leq 1} \underline{F}(r)-F(r(1)) \subset D_{0} \cup N, \\
& \cup_{0 \leq r \leq r(1)} \bar{F}(r)-F(r(1)) \subset D_{\infty} \cup N,
\end{aligned}
$$

further

$$
\begin{aligned}
& D_{0} \subset \cup_{r(1) \leq r \leq 1} \underline{F}(r), \\
& D_{\infty} \subset \cup_{0 \leq r \leq r(1)} \bar{F}(r) .
\end{aligned}
$$


Proof. Let $a<p<1$. From Proposition 1, we clearly see that if $x \in$ $\cup_{0 \leq r \leq r(1)} \bar{F}(r)-F(r(1))$, then $\limsup _{n \rightarrow \infty} \frac{\log \gamma_{p}\left(c_{n}(x)\right)}{\log \left|c_{n}(x)\right|}>1$. This implies that $\liminf _{n \rightarrow \infty} \frac{\gamma_{p}\left(c_{n}(x)\right)}{\left|c_{n}(x)\right|}=0$. From Theorem 9, we see that $x \notin D_{\infty}$, which means $x \in D_{0} \cup N$.

Similarly if $x \in \cup_{r(1) \leq r \leq 1} \underline{F}(r)-F(r(1))$, then $\liminf _{n \rightarrow \infty} \frac{\log \gamma_{p}\left(c_{n}(x)\right)}{\log \left|c_{n}(x)\right|}<$ 1 from Proposition 1. This implies that $\limsup _{n \rightarrow \infty} \frac{\gamma_{p}\left(c_{n}(x)\right)}{\left|c_{n}(x)\right|}=\infty$, which means $x \in D_{\infty} \cup N$ from the above theorem. For $D_{0} \subset \cup_{0 \leq r \leq r(1)} \bar{F}(r)$, we only need to show that $D_{0} \not \subset \underline{F}(r(1))-F(r(1))$ noting Theorem 7 and the above corollary. The contraposition of the above argument implies that if $x \notin D_{\infty} \cup N$, then $x \notin \cup_{r(1) \leq r \leq 1} \underline{F}(r)-F(r(1))$. This means that if $x \in D_{0}$, then $x \notin \underline{F}(r(1))-F(r(1))$. Similarly it follows that $D_{\infty} \subset \cup_{r(1) \leq r \leq 1} \underline{F}(r)$. It follows also for $0<p<a$ from the dual arguments with Theorem 8 .

Remark 4. From Corollary 11, we see that $\underline{F}(0) \cap \bar{F}(1) \subset N$. Since $\underline{F}(0) \cap \bar{F}(1)$ is comeager in $(0,1]([12])$, which means that the base transformed identical code function such that $f\left([\sigma]_{(a)}\right)=[\sigma]_{(p)}$ where $0<p(\neq a)<1$ is nowhere differentiable in the sense of topological magnitude. We also note that its packing dimension $\operatorname{Dim}(\underline{F}(0) \cap \bar{F}(1))=1([3,12])$. We note that if $x$ is an end point of a fundamental interval, then $x \in F(0) \subset \underline{F}(0)$ and $x \in N$.

Remark 5. Since the set of normal points has Lebesgue measure 1 ([9]) and $D_{0}$ has also Lebesgue measure $1, F(a) \cap D_{0}$ has Lebesgue measure 1 . That is, almost all of the points of $F(a)$ are in $D_{0}$ and almost all of the points of $D_{0}$ are in $F(a)$. Further $F(a) \subset D_{0} \cup N$ from the above corollary.

\section{Theorem 13.}

$$
0<g(r(1), r(1)) \leq \operatorname{dim}(N) \leq \operatorname{Dim}(N)=1 .
$$

Further,

$$
\operatorname{dim}\left(D_{\infty}\right) \leq \operatorname{Dim}\left(D_{\infty}\right) \leq g(r(1), r(1))<1 .
$$

Proof. From Corollary 11, $\underline{F}\left(r_{1}\right) \cap \bar{F}\left(r_{2}\right) \subset N$ for every $r_{1}, r_{2}$ such that $0 \leq$ $r_{1}<r(1)<r_{2} \leq 1$, which gives $\operatorname{dim}(N) \geq \inf \left\{g\left(r_{1}, r_{1}\right), g\left(r_{2}, r_{2}\right)\right\}$ for every $r_{1}, r_{2}$ such that $0 \leq r_{1}<r(1)<r_{2} \leq 1$ from $[2,13]$. Hence $\operatorname{dim}(N) \geq$ $g(r(1), r(1))>0 . \operatorname{Dim}(N)=1$ follows from Remark 4. Noting $\operatorname{dim}(E) \leq$ $\operatorname{Dim}(E)$ for every set $E$ and $g(r(1), r(1))<1$, we only need to show that $\operatorname{Dim}\left(D_{\infty}\right) \leq g(r(1), r(1))$. For $a<p<1$, as we argued in the proof of Theorem $7, g(r, p)$ is a decreasing function for $r \in[0,1]$. We note that $\liminf _{k \rightarrow \infty} \frac{n_{0}(x \mid k)}{k}=r \Leftrightarrow \limsup _{k \rightarrow \infty} \frac{\log \gamma_{p}\left(c_{k}(x)\right)}{\log \left|c_{k}(x)\right|}=g(r, p)$ (cf. Lemma 1 in [1]) and $a<r(1)<1$. Hence if $r \geq r(1)$, then $g(r, r(1)) \leq g(r(1), r(1))$. Therefore if $x \in \cup_{r(1) \leq r \leq 1} \underline{F}(r)$, then $\limsup _{k \rightarrow \infty} \frac{\log \gamma_{r(1)}\left(c_{k}(x)\right)}{\log \left|c_{k}(x)\right|} \leq g(r(1), r(1))$, which means that $\operatorname{Dim}\left(\cup_{r(1)} \leq r \leq 1 \underline{F}(r)\right) \leq g(r(1), r(1))$ from the cylinder density theorem in $[2,11]$ (cf. Proposition 2.3 in [10]).

$$
\operatorname{Dim}\left(D_{\infty}\right) \leq g(r(1), r(1))
$$


follows from the above corollary. Dually it holds for $0<p<a$.

\section{Application of the characterizations to $(\tau, \tau-1)$-expansion}

In the above section, we used $f$ for the RNT function, but in this section we will use $\Phi_{\alpha, \tau}(x)$ instead of $f$ for the comparison between our results and those of [14]. J. Paradís et al. ([14]) studied the RNT singular function $\Phi_{\alpha, \tau}(x)$ and gave a critical point to check the existence of derivative of the singular function. The critical point is $K=K(\alpha, \tau)=\frac{\log \left(\frac{\alpha-1}{\tau-1}\right)}{\log (\alpha / \tau)}$. It is not difficult to show that $K=\frac{1}{1-r(1)}$, where $r(1)$ is the solution $r$ of

$$
g(r, p)=\frac{r \log p+(1-r) \log (1-p)}{r \log a+(1-r) \log (1-a)}=1,
$$

where $p=\frac{1}{\tau}$ and $a=\frac{1}{\alpha}$ as it is defined in the previous section. J. Paradís et al. argued in Theorem 4.2 in [14] that for $x \in(0,1]$ where $x=\sum_{j=1}^{\infty} \frac{(\alpha-1)^{j-1}}{\alpha^{a_{j}}}$ with positive integers $a_{j}$ such that $1 \leq a_{1}<a_{2}<\cdots$ if

$$
\liminf _{n \rightarrow \infty} \frac{a_{n}}{n}>K
$$

then, if $\Phi_{\alpha, \tau}^{\prime}(x)$ where $1<\tau<\alpha$ exists in a wide sense, it has to be infinite. The following lemma gives that the above $x \in \underline{F}(r)$ where $r>r(1)=1-\frac{1}{K}$. From the above corollary, we have for $a<p<1, \cup_{r(1) \leq r \leq 1} \underline{F}(r)-F(r(1)) \subset D_{\infty} \cup N$. Putting $a=\frac{1}{\alpha}$ and $p=\frac{1}{\tau}(\Leftrightarrow 1<\tau<\alpha)$, we immediately have their arguments.

Similarly J. Paradís et al. ([14]) argued that for $x \in(0,1]$ where $x=$ $\sum_{j=1}^{\infty} \frac{(\alpha-1)^{j-1}}{\alpha^{a_{j}}}$ with positive integers $a_{j}$ such that $1 \leq a_{1}<a_{2}<\cdots$ if

$$
\limsup _{n \rightarrow \infty} \frac{a_{n}}{n}<K
$$

then, if $\Phi_{\alpha, \tau}^{\prime}(x)$ where $1<\tau<\alpha$ exists in a wide sense, it has to be 0 . In this case, the following lemma gives this $x \in \bar{F}(r)$ where $r<r(1)=1-\frac{1}{K}$. From the result $\cup_{0 \leq r \leq r(1)} \bar{F}(r)-F(r(1)) \subset D_{0} \cup N$ in the above corollary for $a<p<1$, we immediately have their arguments. Similarly we have their arguments for the case $\tau>\alpha>1$ in Theorem 4.2 in [14] using the above corollary.

In the following lemma, we assume that $x=\sum_{j=1}^{\infty} \frac{(\alpha-1)^{j-1}}{\alpha^{a_{j}}}$ with positive integers $a_{j}$ such that $1 \leq a_{1}<a_{2}<\cdots$ as in [14].

Lemma 14. For an extended real number $1 \leq A \leq \infty$ we have

$$
\liminf _{n \rightarrow \infty} \frac{a_{n}}{n} \geq A \Leftrightarrow \liminf _{m \rightarrow \infty} \frac{n_{0}(x \mid m)}{m} \geq 1-\frac{1}{A},
$$

and

$$
\limsup _{n \rightarrow \infty} \frac{a_{n}}{n} \leq A \Leftrightarrow \limsup _{m \rightarrow \infty} \frac{n_{0}(x \mid m)}{m} \leq 1-\frac{1}{A} .
$$


Proof. Suppose that $\liminf _{n \rightarrow \infty} \frac{a_{n}}{n} \geq A$. Consider a positive real number $A^{\prime}$ such that $A^{\prime}<A$. Then there is a positive integer $M$ and a real number $h$ such that $\frac{a_{n}}{n}>h>A^{\prime}$ where $h>A^{\prime}$ for all $n \geq M$. Then for all $n \geq M$,

$$
\frac{n}{a_{n}}<\frac{n}{h n}=\frac{1}{h}<\frac{1}{A^{\prime}} .
$$

For all $n \geq M$ with an integer $i$ such that $0 \leq i<a_{n+1}-a_{n}$,

$$
\frac{n}{a_{n}+i} \leq \frac{n}{a_{n}}<\frac{1}{h}<\frac{1}{A^{\prime}} \text {. }
$$

We note that $x$ has $n$ as the number of times the digit 1 occurs in the first $a_{n}$ places of the code $\sigma(=x)$. Denoting $n_{1}(x \mid m)$ the number of times the digit 1 occurs in the first $m$ places of $\sigma(=x)$ as we denote $n_{0}(x \mid m)$ the number of times the digit 0 occurs in the first $m$ places of $x=\sigma$ in our Preliminaries, we have $\frac{n_{1}(x \mid m)}{m}<\frac{1}{h}<\frac{1}{A^{\prime}}$ for all $m \geq a_{M}(\geq M)$. Hence $\lim \sup _{m \rightarrow \infty} \frac{n_{1}(x \mid m)}{m} \leq \frac{1}{h}<\frac{1}{A^{\prime}}$. Since $n_{0}(x \mid m)=m-n_{1}(x \mid m)$, we have

$$
\liminf _{m \rightarrow \infty} \frac{n_{0}(x \mid m)}{m}=1-\limsup _{m \rightarrow \infty} \frac{n_{1}(x \mid m)}{m} \geq 1-\frac{1}{h}>1-\frac{1}{A^{\prime}} .
$$

Since $A^{\prime}$ is an arbitrary positive real number such that $A^{\prime}<A$, it follows. The converse follows from that the sequence $\left\{\frac{n_{1}(x \mid m)}{m}\right\}$ has $\left\{\frac{n}{a_{n}}\right\}$ as its subsequence. More precisely, if $\liminf \operatorname{in}_{m \rightarrow \infty} \frac{n_{0}(x \mid m)}{m} \geq 1-\frac{1}{A}$, then

$$
\limsup _{m \rightarrow \infty} \frac{n_{1}(x \mid m)}{m} \leq \frac{1}{A} .
$$

Hence $\lim \sup _{n \rightarrow \infty} \frac{n}{a_{n}} \leq \frac{1}{A}$. Therefore $\liminf _{n \rightarrow \infty} \frac{a_{n}}{n} \geq A$.

For the second part, suppose that $\limsup _{n \rightarrow \infty} \frac{a_{n}}{n} \leq A$. We may assume that $1 \leq A<\infty$ since $A=\infty$ gives a trivial case. Consider a real number $A^{\prime}$ such that $A^{\prime}>A$. Then there is a positive integer $M$ and a real number $h$ such that $\frac{a_{n}}{n}<h<A^{\prime}$ where $h<A^{\prime}$ for all $n \geq M$. Then for all $n \geq M$,

$$
\frac{n}{a_{n}}>\frac{n}{h n}=\frac{1}{h}>\frac{1}{A^{\prime}} .
$$

For all $n \geq M$ with an integer $i$ such that $0 \leq i<a_{n+1}-a_{n}$,

$$
\frac{n}{a_{n+1}-i}=\frac{n+1}{a_{n+1}-i}-\frac{1}{a_{n+1}-i}>\frac{n+1}{a_{n+1}}-\frac{1}{a_{n}}>\frac{1}{h}-\frac{1}{a_{n}} .
$$

Since $a_{n} \rightarrow \infty$ as $n \rightarrow \infty$, there is a positive integer $M^{\prime} \geq M$ and a real number $h^{\prime}$ such that $\frac{n}{a_{n+1}-i}>\frac{1}{h^{\prime}}>\frac{1}{A^{\prime}}$ where $h<h^{\prime}<A^{\prime}$ for all $n \geq M^{\prime}$. So $\frac{n_{1}(x \mid m)}{m}>\frac{1}{h^{\prime}}>\frac{1}{A^{\prime}}$ for all $m \geq a_{M^{\prime}}\left(\geq M^{\prime}\right)$. Hence $\liminf \inf _{m \rightarrow \infty} \frac{n_{1}(x \mid m)}{m} \geq \frac{1}{h^{\prime}}>$ $\frac{1}{A^{\prime}}$. Therefore we have

$$
\limsup _{m \rightarrow \infty} \frac{n_{0}(x \mid m)}{m} \leq 1-\frac{1}{h^{\prime}}<1-\frac{1}{A^{\prime}} .
$$


Since $A^{\prime}$ is an arbitrary real number such that $A^{\prime}>A$, it follows. The converse follows from that the sequence $\left\{\frac{n_{1}(x \mid m)}{m}\right\}$ has $\left\{\frac{n}{a_{n}}\right\}$ as its subsequence.

From the above lemma, we easily see that

$$
\liminf _{m \rightarrow \infty} \frac{n_{0}(x \mid m)}{m}=1-\frac{1}{\liminf _{n \rightarrow \infty} \frac{a_{n}}{n}}
$$

and

$$
\limsup _{m \rightarrow \infty} \frac{n_{0}(x \mid m)}{m}=1-\frac{1}{\lim \sup _{n \rightarrow \infty} \frac{a_{n}}{n}} .
$$

As we discussed earlier, the following theorem for the RNT singular function $\Phi_{\alpha, \tau}(x)$ is a generalization of Theorem 4.2 in [14] which is a main result of [14]. From now on, we assume

$$
\Phi_{\alpha, \tau}(x)=\sum_{j=1}^{\infty} \frac{(\tau-1)^{j-1}}{\tau^{a_{j}}}
$$

for

$$
x=\sum_{j=1}^{\infty} \frac{(\alpha-1)^{j-1}}{\alpha^{a_{j}}}
$$

with integers $1 \leq a_{1}<a_{2}<\cdots<a_{n}<\cdots$ with $\alpha \neq \tau$ where $\alpha, \tau>1$ and $K=K(\alpha, \tau)=\frac{\log \left(\frac{\alpha-1}{\tau-1}\right)}{\log (\alpha / \tau)}$. We easily see that $K(\alpha, \tau) \geq 1$.

Theorem 15. If $\lim _{n \rightarrow \infty} \frac{a_{n}}{n} \neq K$, then we have:

(i) Case $1<\tau<\alpha$. If

$$
\limsup _{n \rightarrow \infty} \frac{a_{n}}{n} \leq K,
$$

then, if $\Phi_{\alpha, \tau}^{\prime}(x)$ exists in a wide sense, it has to be 0. If

$$
\liminf _{n \rightarrow \infty} \frac{a_{n}}{n} \geq K
$$

then, if $\Phi_{\alpha, \tau}^{\prime}(x)$ exists in a wide sense, it has to be $\infty$.

(ii) Case $\tau>\alpha>1$.

$$
\liminf _{n \rightarrow \infty} \frac{a_{n}}{n} \geq K
$$

then, if $\Phi_{\alpha, \tau}^{\prime}(x)$ exists in a wide sense, it has to be 0. If

$$
\limsup _{n \rightarrow \infty} \frac{a_{n}}{n} \leq K
$$

then, if $\Phi_{\alpha, \tau}^{\prime}(x)$ exists in a wide sense, it has to be $\infty$.

Proof. For Case $1<\tau<\alpha$, assume that $\liminf _{n \rightarrow \infty} \frac{a_{n}}{n} \geq K$. The above lemma gives that

$$
\liminf _{m \rightarrow \infty} \frac{n_{0}(x \mid m)}{m} \geq 1-\frac{1}{K}=r(1) .
$$

Since $\lim _{n \rightarrow \infty} \frac{a_{n}}{n} \neq K$, we easily see that $x \notin F(r(1))$ from the above lemma. From $\cup_{r(1) \leq r \leq 1} \underline{F}(r)-F(r(1)) \subset D_{\infty} \cup N$ which is a result of Corollary 12 for 
$a<p<1$, we immediately have $\Phi_{\alpha, \tau}^{\prime}(x)=\infty$ if $\Phi_{\alpha, \tau}^{\prime}(x)$ exists in a wide sense. The rest follows from similar arguments.

The following theorem is the converse of the above theorem in some sense, which was not mentioned in [14].

Theorem 16. We have:

(i) Case $1<\tau<\alpha$. If $\Phi_{\alpha, \tau}^{\prime}(x)=0$, then

$$
\limsup _{n \rightarrow \infty} \frac{a_{n}}{n} \leq K \text {. }
$$

If $\Phi_{\alpha, \tau}^{\prime}(x)=\infty$, then

$$
\liminf _{n \rightarrow \infty} \frac{a_{n}}{n} \geq K
$$

(ii) Case $\tau>\alpha>1$. If $\Phi_{\alpha, \tau}^{\prime}(x)=0$, then

$$
\liminf _{n \rightarrow \infty} \frac{a_{n}}{n} \geq K
$$

If $\Phi_{\alpha, \tau}^{\prime}(x)=\infty$, then

$$
\limsup _{n \rightarrow \infty} \frac{a_{n}}{n} \leq K
$$

Proof. It follows from the above lemma with the results of Corollary 12 which are not used in the proof of the above theorem.

The following theorem is a new fact for a sufficient condition for the nondifferentiability points of the RNT singular distribution.

Theorem 17. If $\lim \inf _{n \rightarrow \infty} \frac{a_{n}}{n}<K<\limsup _{n \rightarrow \infty} \frac{a_{n}}{n}$, then $\Phi_{\alpha, \tau}^{\prime}(x)$ does not exist.

Proof. From the above lemma, if $\liminf _{n \rightarrow \infty} \frac{a_{n}}{n}<K<\limsup _{n \rightarrow \infty} \frac{a_{n}}{n}$, then we easily see that

$$
x \in\left[\cup_{r(1)<r \leq 1} \bar{F}(r)\right] \cap\left[\cup_{0 \leq r<r(1)} \underline{F}(r)\right] .
$$

It follows from Corollary 11.

The following theorem is also an essential generalization of Theorem 4.1 in [14]. It assures that $\Phi_{\alpha, \tau}^{\prime}(x)=0$ for a normal point $x \in F_{a}$ where $a=\frac{1}{\alpha}$ when $\Phi_{\alpha, \tau}^{\prime}(x)$ exists in a wide sense.

Theorem 18. If $\lim _{n \rightarrow \infty} \frac{a_{n}}{n}=\frac{\alpha}{\alpha-1}$, then, if $\Phi_{\alpha, \tau}^{\prime}(x)$ exists in a wide sense, $\Phi_{\alpha, \tau}^{\prime}(x)=0$.

Proof. If $\lim _{n \rightarrow \infty} \frac{a_{n}}{n}=\frac{\alpha}{\alpha-1}$, then $x \in F\left(\frac{1}{\alpha}\right)$ from the above lemma. From $F\left(\frac{1}{\alpha}\right)=F(a) \subset D_{0} \cup N$ in Remark 5 , it follows. 


\section{References}

[1] I. S. Baek, Relation between spectral classes of a self-similar Cantor set, J. Math. Anal. Appl. 292 (2004), no. 1, 294-302.

[2] Dimensions of distribution sets in the unit interval, Commun. Korean Math. Soc. 22 (2007), no. 4, 547-552.

[3] I. S. Baek, L. Olsen, and N. Snigireva, Divergence points of self-similar measures and packing dimension, Adv. Math. 214 (2007), no. 1, 267-287.

[4] P. Billingsley, Probability and Measure, John Wiley \& Sons, Inc., New York, 1995.

[5] C. D. Cutler, A note on equivalent interval covering systems for Hausdorff dimension on $R$, Internat. J. Math. Math. Sci. 11 (1988), no. 4, 643-649.

[6] R. Darst, The Hausdorff dimension of the nondifferentiability set of the Cantor function is $[\ln (2) / \ln (3)]^{2}$, Proc. Amer. Math. Soc. 119 (1993), no. 1, 105-108.

[7] _ Hausdorff dimension of sets of non-differentiability points of Cantor functions, Math. Proc. Cambridge Philos. Soc. 117 (1995), no. 1, 185-191.

[8] J. Eidswick. A characterization of the nondifferentiability set of the Cantor function, Proc. Amer. Math. Soc. 42 (1974), 214-217.

[9] K. J. Falconer, The Geometry of Fractal Sets, Cambridge University Press, Cambridge, 1986.

[10] _ Techniques in Fractal Geometry, John Wiley \& Sons, Ltd., Chichester, 1997.

[11] H. H. Lee and I. S. Baek, A note on equivalent interval covering systems for packing dimension of $R$, J. Korean Math. Soc. 28 (1991), no. 2, 195-205.

[12] L. Olsen, Extremely non-normal numbers, Math. Proc. Cambridge Philos. Soc. 137 (2004), no. 1, 43-53.

[13] L. Olsen and S. Winter, Normal and non-normal points of self-similar sets and divergence points of self-similar measures, J. London Math. Soc. (2) 67 (2003), no. 1, 103-122.

[14] J. Paradís, P. Viader, and L. Bibiloni, Riesz-Nágy singular functions revisited, J. Math. Anal. Appl. 329 (2007), no. 1, 592-602.

[15] H. L. Royden, Real Analysis, Macmillan Publishing Company, 1988.

Department of Mathematics

Pusan University of Foreign Studies

PUsAn 608-738, KoreA

E-mail address: isbaek@pufs.ac.kr 